\section{Deep common bile duct cannulation time at endoscopic retrograde cholangio- pancreatography: a forgotten parameter for assessment of endoscopic competence?}

\author{
Mohammad Wehbi, Emad Qayed, Tanvi \\ Dhere, Chetan Gondha, M. Kamil \\ Obideen, Qiang Cai \\ Division of Digestive Diseases, Emory \\ University, School of Medicine, Atlanta, \\ GA, USA
}

\section{Abstract}

The rate of successful deep common bile duct cannulation (DCBD) at endoscopic retrograde cholangiopancreatography (ERCP) is usually used as a surrogate marker of competence at ERCP. There are few data regarding the time spent on cannulation at ERCP. This prospective study aimed to evaluate the time spent on DCBD cannulation at ERCP and to provide a rationale for establishing the DCBD cannulation time as another parameter in assessment of ERCP competence. This is a prospective study performed in a single tertiary university-based referral center. DCBD cannulation time as well as the fluorescence time and the cost of cannulation tools during DCBD cannulation were measured. The mean DCBD cannulation was $12.5 \pm 13.6$ minutes. Eighty-percent of the cannulation was achieved within 10 min, $10 \%$ achieved in 10-30 min, and the remaining in longer than $30 \mathrm{~min}$. The longer cannulation time was associated with increased the cost of cannulation (\$79/cannulation versus \$387/ cannulation, $\mathrm{P}<0.001$ ), as well as increasing the radiation exposure times (3.1 $\mathrm{min} /$ cannulation vs. 25 $\mathrm{min} /$ cannulation, $\mathrm{P}<0.001)$. In addition to the success rate of DCBD cannulation, the DCBD cannulation time should be considered as another parameter in the assessment of endoscopic competence in ERCP.

\section{Introduction}

Endoscopic retrograde cholangiopancreatography (ERCP) is an advanced endoscopic procedure that has been used in clinical practice for more than three decades. ${ }^{1,2}$ It is commonly used for the diagnosis and treatment of biliary and pancreatic diseases.$^{1.5}$ ERCP is a technically demanding endoscopic procedure requiring a considerable amount of training to be performed safely. ${ }^{6}$ A key step to complete the procedure is to cannulate the common bile duct (CBD) and/or pancreatic duct. Deep cannulation of the CBD at ERCP can represent a technical challenge, even to experienced pancreaticobiliary endoscopists. ${ }^{7,}$ In fact, the most common reason for an unsuccessful ERCP is the inability to cannulate the CBD. ${ }^{7,8}$

Trainee endoscopists require extensive supervision and hands-on experience to develop expertise to perform the procedure safely and effectively. ${ }^{9-15}$ The development of procedural competence and achievement of threshold success rates have been positively correlated to the number of procedures performed under supervision. ${ }^{9-15}$ Studies have suggested that to attain basic competence, a trainee should achieve an overall $80 \%$ likelihood of successful ERCP cannulation. ${ }^{9,12}$

The previously published studies emphasized only the successful cannulation rate but not the time spent on cannulation. DCBD cannulation is a crucial step in ERCP and is a prerequisite to the performance of therapeutic interventions, such as stone removal and stent placement. The success rate for DCBD cannulation at ERCP should be an important assessment for the procedural competence. We also believe that the amount of time spent in DCBD cannulation should be considered as another parameter to assess competence.

\section{Materials and Methods}

\section{Patients}

A prospective study was conducted at Emory University Hospital. Patients referred to Emory University Hospital for an ERCP procedure were assessed for eligibility of enrollment over a 10-month period. The exclusion criteria for this study were: i) history of endoscopic or surgical sphincterotomy; ii) pre-existing stent in CBD or PD; iii) having had ERCP within one week prior to the study; iv) age younger than 18 years old; v) pregnancy. Enrolled patients signed a written consent.

\section{Endoscopists}

Two experienced pancreaticobiliary endoscopists (attendings) performed the ERCP procedures. Both of them had performed more than 1,000 ERCPs. As the study was performed at a tertiary teaching hospital, four ERCP fellows (trainees) performed the procedure together with one of the attendings. The trainees were third-year gastroenterology fellows. Each trainee had already performed 50 or so supervised ERCP procedures at the time of this study. The procedures were started by the trainee. After appropriate positioning of the endoscopes was achieved, the trainee had five
Correspondence: Qiang Cai, Division of Digestive Diseases, 1365 Clifton Road, B1262,

Emory University School of Medicine, Atlanta, GA 30322, USA. E-mail: qcai@emory.edu

Key words: endoscopic retrograde cholangiopancreatography, cannulation time, fluoroscopy time, endoscopic retrograde cholangiopancreatography competence.

Received for publication: 28 January, 2010.

Revision received: 7 April 2010.

Accepted for publication: 5 May 2010.

This work is licensed under a Creative Commons Attribution 3.0 License (by-nc 3.0).

(C) Copyright M. Wehbi et al., 2010

Licensee PAGEPress, Italy

Gastroenterology Insights 2010; 2:e7

doi:10.4081/gi.2010.e7

minutes for cannulation. If DCBD cannulation was successfully achieved within five minutes, the trainee was allowed to continue the procedure under the guidance of the attending. If cannulation of the CBD could not be achieved in five minutes, an attending took over the endoscope and continued the cannulation. After successful cannulation was achieved by the attending, the trainee was allowed to continue the procedure. The decision to intervene by the attending was based on whether or not the maneuvers performed by the trainee were deemed appropriate and likely to be successful in the rest of the procedure. In terms of equipment used for cannulation, one attending often used a standard catheter, whereas the other started with a ball-tipped catheter. A sphincterotome or a guide wire to assist cannulation was also used when necessary.

\section{Definitions}

Procedural time: i) pre-cannulation time was defined as the time from esophageal intubation to the time of appropriate positioning of the endoscope proximal to the ampulla prior to cannulation; ii) DCBD cannulation time was defined as the time from the initiation of cannulation to the time when the catheter was deep inside the CBD at which time therapeutic interventions could be performed; iii) post cannulation time was defined as the time from the end of cannulation to the time when the procedure was completed; iv) total procedure time was defined as the time from the esophageal intubation to the time of withdrawal of the endoscope from the patient, which was the sum of the above three times.

When recording the above times, the counting was continuous without any interruptions. For example, if a trainee took five minutes for cannulation and an attending took two min- 
utes to achieve a DCBD cannulation, the total DCBD cannulation time was recorded as seven minutes. The percent of cannulation was calculated in the same way.

Fluoroscopy time (our fluoroscopy machine automatically showed the fluoroscopy used in minutes at each interval): i) cannulation fluoroscopy time was recorded as the fluoroscopy used in minutes from the beginning of the procedure to the end of the cannulation; ii) postcannulation fluoroscopy time was recorded as the fluoroscopy used in minutes from the end of the cannulation to the completion of the procedure; iii) total fluoroscopy time was recorded as the fluoroscopy used in minutes from the beginning to the end of the procedure, which included the fluoroscopy time used during cannulation plus the time used during post-cannulation. Failed cannulation was defined as not being able to achieve successful cannulation in $60 \mathrm{~min}$. In addition, the equipment, such as the catheters and guide wires used for each cannulation were recorded. The cost for each catheter and guide wires used during each cannulation were calculated. Patients were followed up once next day or longer if needed for possible complications.

\section{Research nurse}

A research nurse was present during each procedure in the study. The research nurse precisely recorded the different times and the equipment used during each cannulation.

\section{Statistical analysis}

The Spearman rank correlation coefficient (rs) was used to determine the association between DCBD cannulation time, cannulation cost and radiation time. ${ }^{16}$ The difference with a $P$ value less than 0.05 was considered statistically significant.

\section{Results}

Two hundred and twelve patients were screened for the study (Figure 1). Of these, 102 patients were eligible to be enrolled in the study. There was a $92 \%$ successful cannulation rate. Of the 94 patients that were successfully cannulated, 4 became unstable during the procedure resulting in early termination of the procedure. The remaining 90 patients completed the procedure. The study population included 38 females and 52 males with a mean age of 58 years. Seventy-six ERCPs were performed with some interventions and 14 were performed without any interventions (diagnostic ERCP) (Figure 1). These 14 patients had abnormal LFT and abnormal images, but their cholangiogram were normal on ERCP.

\section{Procedural times}

The average pre-cannulation time was 6.5 min, DCBD cannulation time was $12.2 \mathrm{~min}$, and the post-cannulation time was $21.2 \mathrm{~min}$. The average time to complete an ERCP procedure in this study was 40 min (Table 1).

The trainee cannulated 35 patients within five minutes and the attending cannulated another 37 patients in the next five minutes. Therefore, DCBD cannulation was achieved in 72 patients $(80 \%)$ within ten minutes. Of the remaining 18 patients, DCBD cannulation was achieved in 10-30 min in 9 patients and over 30 min in the other 9 patients (Table 2).

Eight patients failed cannulation in this study; their procedures were terminated after attempts were made for $60 \mathrm{~min}$.

\section{Fluoroscopy time}

The average fluoroscopy time used during cannulation was $5.8 \mathrm{~min}$, and in post-cannulation was $7.7 \mathrm{~min}$. The average total fluoroscopy used was $13.4 \mathrm{~min}$. The fluoroscopy time was significantly longer if cannulation was not achieved efficiently (Table 3 ).

\section{Costs of materials during cannulation}

In the 72 patients whom the CBD was cannulated within ten minutes, only one catheter was used during the cannulation; each of the standard or ball-tipped catheters costs the same at $\$ 79$. In the remaining 18 patients, more than one catheter and guide wires were used in some patients; a sphincterotome catheter costs $\$ 170$ each. The cost for cannula-

Table 1. ERCP procedural and fluoroscopy times (minutes, mean $\pm S D, n=90$ ).

\begin{tabular}{cccc}
\hline Pre-cannulation time & Cannulation time* & Post-cannulation time & Total time \\
$6.5 \pm 5.6$ & $12.2 \pm 13.6$ & $21.2 \pm 14.5$ & $40.0 \pm 20.0$ \\
Fluoroscopy on cannulation & Fluoroscopy on post-cannulation & Total fluoroscopy \\
$5.8 \pm 7.3$ & $7.7 \pm 9.6$ & $13.4 \pm 14.1$ \\
\hline
\end{tabular}

*Including trainee's cannulation time, 5 min.

Table 2. Efficacy of DCBD cannulation $(n=90)$.

\begin{tabular}{|c|c|c|}
\hline DCBD cannulation time (min) * & N. patients & $\%$ \\
\hline$<10$ & 72 & 80 \\
\hline 10 to 30 & 9 & 10 \\
\hline$>30$ & 9 & 10 \\
\hline
\end{tabular}

* Including trainee's cannulation time, 5 min.

Table 3. Comparison of cost and radiation exposure during cannulation.

$\begin{array}{lcccc}\text { DCBD cannulation time }(\min )^{*} & <10 & 10 \text { to } 30 & >30 & \text { P } \\ \text { Cost for cannulation/patient } & 79.0 \pm 0.0 & 207.9 \pm 106.7 & 387.9 \pm 67.5 & <0.001 \\ \text { Fluoroscopy time/patient } & 3.1 \pm 2.0 & 7.7 \pm 3.0 & 25.5 \pm 6.0 & <0.001\end{array}$

*Including trainee's cannulation time, 5 min.

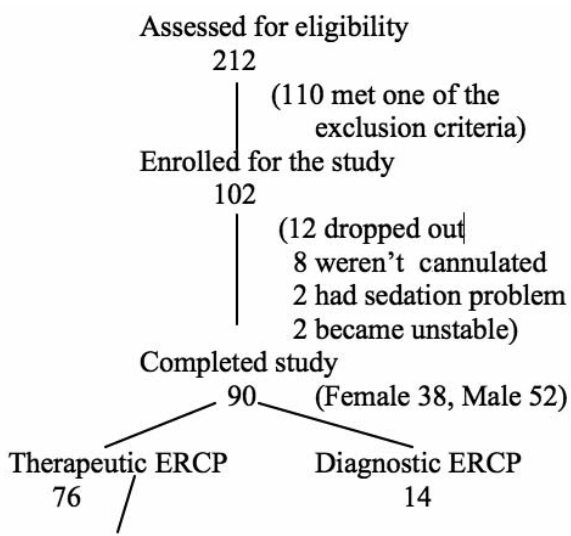

45: benign stricture in the common bile duct

11: malignant stricture in the common bile duct

20: stones in the common bile duct

(Of the 76, 13 patients had failed ERCP from outside facility)

Figure 1. Study profile. 
tion material was significantly higher when cannulation was not achieved efficiently (Table 3). Fifteen patients had some abdominal pain after the procedure, but none of them met the criteria for post-ERCP pancreatitis, since the pain was resolved within a few hours. There were no other complications associated with this study.

\section{Discussion}

Ensuring competence in ERCP has always been a focus of interest as the procedure has one of the highest complication rates among the procedures routinely performed by gastrointestinal endoscopists. The estimated morbidity and mortality are $5-10 \%$ and $0.1-1 \%$, respectively. ${ }^{15,17-20}$ The rate of complications is related to several factors including the indications for the procedure and the endoscopic technique. ${ }^{15,17-20}$

There is no doubt that successful cannulation at ERCP is very important. In the era of magnetic resonance cholangiopancretogram (MRCP), ERCP is more often used for thera-

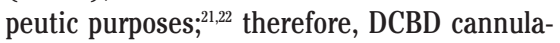
tion, as a prerequisite for therapeutic intervention, is more important than in the past. Indeed, the most common reason for an unsuccessful ERCP is the inability to cannulate the CBD ${ }^{7,8}$ However, a prolonged procedure related to factors such as difficult cannulation is thought to be a predictive factor of an increased rate of complications after ERCP. ${ }^{15}$ Therefore, an ideal cannulation would not only be to deep cannulate the common bile duct, but also to cannulate it safely and in a timely manner. Our study suggests that DCBD cannulation time may be an ideal marker to assess an endoscopist's efficacy in cannulation.

The safe and effective practice of ERCP, especially for therapeutic reasons, requires rigorous training and practice, with outcomes dependent on the endoscopist's experience. ${ }^{12}$ Because of the lack of accurate markers for assessing the acquisition of competence during training, the number of supervised procedures needed to attain an acceptable success rate in cannulation has been used as a surrogate marker for objectively assessing learning. ${ }^{911,12,23}$ At present, at least 180-200 supervised procedures have been recommended for a trainee to achieve an overall $80 \%$ likelihood of successful ERCP cannulation. ${ }^{9}$ The recommendation was based on a study that used mixed groups of patients with native anatomy and those with prior sphincterotomy. Deep biliary cannulation is considered more difficult in patients with a native papilla in comparison with those with a prior biliary sphincterotomy. ${ }^{12}$ Therefore, the current recommendation was criticized. ${ }^{12}$ In order to achieve $80 \%$ or more success at deep biliary cannulation in patients with native papillary anatomy, a trainee may need to perform $350-400$ supervised procedures. ${ }^{12}$

However, successful cannulation rate was used as the only marker to assess the trainee's competence in mastering the ERCP procedure in the above studies. There is no doubt that a successful cannulation is the most important step in an ERCP procedure, but use of the successful cannulation rate alone as the only marker to assess the competence is not comprehensive. Our study clearly demonstrated that an efficient cannulation could reduce procedure costs and decrease the radiation exposure during the procedure. Therefore, in addition to the successful cannulation rate, DCBD cannulation time should be considered as another marker to assess the trainee's competence in performing ERCP.

To our knowledge, this is the first study providing detailed information on DCBD cannulation time. The study demonstrated that $80 \%$ of the successful DCBD cannulations on patients with native papilla could be achieved within ten minutes in experienced hands, even though the procedure was performed with trainees. Although we did not see any complications in patients with an inefficient cannulation at ERCP, the results in this study demonstrated that an inefficient cannulation at ERCP was associated with increased procedure costs as well as unnecessary radiation exposure during the cannulation.

The results in this study conclusively verified that DCBD cannulation at ERCP could be achieved in more than $90 \%$ of patients. The results also demonstrated that DCBD cannulation could be achieved within ten minutes in $80 \%$ of patients. We are using an $80 \%$ successful cannulation rate as a marker for assessment of competence. ${ }^{9}$ It is not unknown whether we should use $80 \%$ successful cannulation within ten minutes as another marker for assessment of competence, since the data obtained in this study was obtained from only two endoscopists. Also, in this study, each trainee had five minutes for cannulation; the actual cannulation time may be shorter than ten minutes if performed by the attending alone. In a reviewing of medical literature, there is little information about cannulation time. We previously reported a fatty meal ingested shortly before the ERCP procedure could shorten the DCBD cannulation time. ${ }^{24}$ The average DCBD cannulation time in the present study was comparable to the data in that study.

We believe that ensuring competence for ERCP should not only be based on successful cannulation rate but cannulation time as well. Further studies are needed to evaluate the cannulation time as a competence marker for trainees performing ERCP.

\section{References}

1. Cotton PB. Cannulation of papilla of Vater by endoscopy and retrograde cholangiopancreatography. Gut 1972;13:1014-25.

2. Cotton PB. ERCP. Gut 1977;18:316-41.

3. Sherman S, Lehman GA. Endoscopic retrograde cholangiopancreatography, endoscopic sphincterotomy and stone removal, and endoscopic biliary and pancreatic drainage. In Textbook of Gastroenterology. Yamada T, et al. Third Edition, Lippincott Williams and Wilkins, Philadelphia, New York, Baltimore. 1999;2718-46.

4. National Institutes of Health, State-of Science Conference Statement: Endoscopic retrograde cholangiopancreatography for diagnosis and therapy. 2002.

5. Cotton PB, Williams CB. Practical gastrointestinal endoscopy, 3rd edition ed.

6. Leung JW, Chung RS. Training in ERCP. Gastrointest Endosc 1992;38:517-9.

7. Kumar S, Sherman S, Hawes RH, et al. Success and yield of second attempt ERCP. Gastrointest Endosc 1995;41:445-7.

8. Foutch PG. A prospective assessement of results for needle-knife papilloltomy and standard endoscopic sphincterotomy. Gastrointest Endosc 1995;41:25-32.

9. Jowell PS, Baillie J, Branch S, et al. Quantitative assessment of procedural competence: A prospective study of training in endoscopic retrograde cholangiopancreatography. Ann Intern Med 1996; 125:983-9.

10. Wicks ACB, Robertson GSM, Veitch PS. Structured training and assessment in ERCP has become essential for the Calman era. Gut 1999;45:154-6.

11. Ragunath K, Thomas LA, Cheung WY, et al. Objective evaluation of ERCP procedures: a simple grading scale for evaluating technical difficulty. Postgrad Med J 2003;79: 467-70.

12. Verma D, Gostout CJ, Petersen BT, et al. Establishing a true assessment of endoscopic competence in ERCP during training and beyond: a single-operator learning curve for deep biliary cannulation in patients with native papillary anatomy. Gastrointest Endosc 2007;65:394-400.

13. Peterson BT. ERCP outcomes: defining the operators, experience, and environments. Gastrointest Endosc 2002;55:953-8.

14. Clinical competence in diagnostic endoscopic retrograde cholangiopancreatography. Health and Public Committee, American College of Physicians. Ann Intern Med. 1988;108:142-4.

15. Schutz SM, Abbott RM. Grading ERCPs by degree of difficulty: a new concept to produce more meaningful outcome data. Gastrointest Endosc 2000;51:535-9. 
16. Rosner B. Fundamentals of Biostatistics. 5th edn. Pacific Grove, California: Duxbury Press, 2000.

17. Sultan S, Baillie J. What are the predictors of post-ERCP pancreatitis, and how useful are they? JOP 2002;3:188-94.

18. Standards of Practice Committee. Complications of ECP. Gastrointest Endosc 2003;57:633-8.

19. Vandervoort J, Soetikno RM, Tham TC, et al. Risk factors for complications after per- formance of ERCP. Gastrointest Endosc 2002;56:652-6.

20. Baillie J. Predicting and preventing postERCP pancreatitis. Curr Gastro Rep 2002; 4:112-9.

21. Fayad LM, Kowalski T, Mitchell DG. MR cholangiopancreatography: evaluation of common pancreatic diseases. Rad Clin N Am 2003;41:97-114.

22. Motohara T, Semelka RC, Bader T. MR cholangiopancretography. Rad Clin N Am
2003;41:89-96.

23. Baillie J, Ravich WJ. On endoscopic training and procedural competence. Ann Intern Med 1993;118:73-4.

24. Barrie M, Klein SD, Brown CA, et al. Endoscopic retrograde cholangopancreatography after a liquid fatty meal: Effect on deep common bile duct cannulation time. Endoscopy 2006;38:241-8. 